\title{
Meningitis in Children: A Study in Medical College \& Hospital, Kolkata
}

\author{
SWARNALI JOARDAR ${ }^{1}$, GAUTAM KUMAR JOARDAR ${ }^{2}$, PANKAJ KUMAR MANDAL ${ }^{3}$, SIDDHARTHAMANI ${ }^{4}$
}

\begin{abstract}
Background: Meningitis in children constitutes a formidable illness worldwide due to its high morbidity and mortality. The etiological agents and other associated epidemiological factors contributing to its occurrence and prognosis varies from place to place. Identification of these factors is crucial for the reduction of this important health problem. This record-based observational study was conducted in Medical College \& Hospital, Kolkata, India to identify the socio-demographic profiles, etiological types and nutritional status of the meningitis cases admitted in the Pediatric Medicine department in this institute during the last three years and to find out the duration of hospital stay, case fatality rates and seasonal variations in different etiological types of the disease.

Materials and Method: This observational record based study was conducted in
Medical College \& Hospital, Kolkata, a tertiary care hospital located in central area of
the city of Kolkata, India. It provides health care services to the urban population as
well as rural population of different districts of the state of West Bengal, and also to
population from outside of the state. The available records of all the meningitis cases
admitted in pediatric medicine department of the Institution from January 2007 to
December 2009 were studied. The variables included were age, sex, religion, residence,
nutritional status, etiological type of meningitis, duration of hospital stay, case fatality
rate (CFR) and seasonal variation. The collected data were analyzed with suitable
statistical methods.

Results: A total of 326 meningitis cases in the age group below twelve years comprised the study population. More than $27 \%$ of the cases were infants and about $44 \%$ were below three years of age. The majority of the cases were male (60.43\%), Hindu $(64.42 \%)$ by religion and rural $(74.23 \%)$ by residence. Fifty seven percent of the cases were tuberculous and $34.66 \%$ were pyogenic by etiological type. Among those who survived (224) the overall prevalence of undernutrition was $66.52 \%$ with a significantly higher proportion of undernutrition observed in tuberculous meningitis (75.59\%) compared to that among pyogenic meningitis (54.16\%). For $59.06 \%$ of tuberculous and $57.32 \%$ of pyogenic cases the hospital stay was between 1-2 weeks. The overall case fatality rate (CFR) was $31.29 \%$. Compared to viral meningitis, the CFR was significantly higher both in pyogenic and tuberculous meningitis. Some seasonal variation was also observed with two peaks in case of pyogenic and four peaks in case of tuberculous meningitis.

Conclusion: It can be concluded that case fatality rate was quite high in pyogenic and tuberculous meningitis even in this era of modern medicine. Higher proportion of patients from rural locations and a high prevalence of undernutrition among the cases point to socio-economic aspects of the disease.
\end{abstract}

Key words: Meningitis, Undernutrition, Case fatality rate, Seasonal variation.

1. PGT (MD Pediatrics), Dept. of Pediatric Medicine, Medical College, 88, College Street, Kolkata-700 073

2. Professor, Dept. of Community Medicine, NRS Medical College, 138 AJC Bose Road, Kolkata 700014

3. Associate Professor, Dept. of Community Medicine, Medical College, 88, College Street.

4. Dept. of Medicine, MD Medicine, Medical College, 88 , College Street, Kolkata-700 073

Correspondence: Dr. Gautam Kumar Joardar, Email: gkjoardar@rediffmail.com

\section{Introduction}

The word 'meningitis' literally means 'Inflammation of the meninges'. It is mostly caused due to infection by agents like bacteria, virus, fungi or protozoa. Acute meningitis may be caused by bacteria or virus; whereas, sub-acute or chronic meningitis are usually caused by mycobacterium tuberculosis, fungi or 
parasites. Viral meningitis is rarely severe and children tend to have a complete recovery, whereas bacterial meningitis can have a rapid onset, leading to death and serious neurological sequele ${ }^{1}$. The global burden of the disease is high. Apart from epidemics, at least 1.2 million cases of meningitis are estimated to occur every year with 135000 deaths ${ }^{2}$. Incidence of bacterial meningitis is quite high despite improvement of treatment ${ }^{3}$ and the great majority of cases occur under the age of 5 years; infants being particularly vulnerable ${ }^{4}$. Lack of proper diagnostic facilities, difficulties in culturing the organism, lack of awareness among the people and indiscriminate use of antibiotics may lead to wrong estimation of the true incidence of the actual disease burden. Various factors that determine the outcome of the disease are age, time taken in diagnosis \& initiation of treatment, duration of treatment and type of microorganism ${ }^{5}$.

Incidence of meningitis may vary from place to place and from country to country due to population susceptibility, introduction of new strains and different environmental, socio-demographic and immunological factors. Understanding the relationship between these factors and the disease is the key for identification of high risk groups/areas and thus in taking specific strategies to prevent its occurrence and reduce morbidity and mortality. So the present study was undertaken with the following objectives:

To identify the socio-demographic profiles, etiological types and nutritional status of the meningitis cases admitted in Pediatric Medicine department of Medical College \& Hospital, Kolkata during the last three years (January 2008 to December 2010) and to find out the duration of hospital stay, case fatality rates and seasonal variations in different etiological types of meningitis.

\section{Materials and methods}

This observational record based study was conducted in Medical College \& Hospital, Kolkata, a tertiary care hospital located in central area of the city of Kolkata, India.

It provides health care services to the population of different districts of the state of West Bengal, and also to population from outside of the state. The records of the diagnosed meningitis cases admitted in the Pediatric Medicine department, from January 2007 to December 2009 (preserved in the records section) were studied. The diagnosis of meningitis was confirmed by evaluating the records of CSF examination, culture and/or other relevant assays done during their hospital stay. The records of all such cases were included in the study. Tools used were pre-designed and pre-tested schedule and weight for age chart for Indian children 6,7 . The variables included were age, sex, religion, area of residence (rural / urban), nutritional status, etiological types of meningitis, duration of hospital stay, case fatality rate and seasonal variation. After obtaining ethical clearance from the institutional ethics committee, the registrar of the hospital record section was communicated and all the available records of the meningitis cases were studied. The cases that were admitted and discharged from the hospital as cured or discharged on risk bonds were recorded in a set of registers; whereas, the information about the cases that died after admission were recorded in separate death registers. Nutritional status of the children was assessed by using the weight for age criterion. In this study undernutrition was defined as weight for age of a child less than $3^{\text {rd }}$ percentile of the sex specific weight for age of Indian children $6,7,8$. The data collected were analyzed and tabulated using frequency distribution tables as proportions/ percentages and suitable diagrams. Suitable statistical tests were applied wherever applicable.

\section{Results:}

The records of 326 meningitis cases, in the age group from 0 to 12 years (admitted in the last three years) were studied. The total number of cases admitted (all types) is much more (154) in the year 2008 compared to 2009 (114) and 2010 (58).

Table - I shows that out of 326 cases 197 (60.43\%) were male. By age group 91 (27.91\%) were infants; $184(56.44 \%)$ belonged to age group under five years; $144(44.17 \%)$ belonged to under three years; and 110 (33.74\%) belonged to age of 5-9 years. By religion, $210(64.42 \%)$ were Hindu; and by residence 242 $(74.23 \%)$ were from rural areas. 
Table-I

Socio-demographic profiles of the study population: $(n=326)$

A. Distribution by age and sex

\begin{tabular}{lcccc}
\hline Age & \multicolumn{4}{c}{ Sex } \\
& Male $(n=197)$ & Female $(n=129)$ \\
& No & $\%$ & No & $\%$ \\
\hline 0-11 months $(n=91)$ & 56 & 17.18 & 35 & 10.74 \\
12-23 months $(n=32)$ & 16 & 4.91 & 16 & 4.91 \\
24-35 months $(n=21)$ & 14 & 4.29 & 7 & 2.15 \\
36-47 months $(n=31)$ & 22 & 6.75 & 9 & 2.76 \\
48-59 months $(n=9)$ & 4 & 1.23 & 5 & 1.53 \\
5-9 years $(n=110)$ & 66 & 20.24 & 44 & 13.49 \\
10-12 years $(n=32)$ & 19 & 5.83 & 13 & 3.99 \\
\hline
\end{tabular}

B. Distribution by religion

\begin{tabular}{lcc}
\hline Religion & No & $\%$ \\
\hline Hindu & 210 & 64.42 \\
Muslim & 116 & 35.58 \\
\hline
\end{tabular}

C. Distribution by residence

\begin{tabular}{lcc}
\hline Residence & No & $\%$ \\
\hline Rural & 242 & 74.23 \\
Urban & 84 & 25.77 \\
\hline
\end{tabular}

Table - II shows that among all the meningitis cases in the last three years, the majority $(57.37 \%)$ was tuberculous and $34.66 \%$ were pyogenic by etiology. The viral/aseptic type of meningitis comprised only $7.97 \%$ of cases. Among all cases, the proportion of tuberculosis remained highest throughout.

Out of the total 326 cases of meningitis, 102 children died after admission and the record of their body weights was not available. So, the nutritional status was assessed for the 224 survivors.

Table - III shows that among the tuberculous meningitis cases, $75.59 \%$ cases were undernourished. Among the other types, undernutrition was present in $54.16 \%$ of the pyogenic and $56.00 \%$ of the viral/aseptic cases. It is evident that proportion of undernutrition among tuberculous meningitis (TBM) cases was significantly higher than that among pyogenic meningitis cases $(p<0.01)$. Overall prevalence of undernutrition was $66.52 \%$.

Table - IV shows that in $59.06 \%$ of the tuberculous and $57.32 \%$ of pyogenic meningitis cases duration of hospital stay was between $1-2$ weeks duration. Hospital stay for more than two weeks was proportionately much higher in tuberculous type ( $26.77 \%)$ compared to pyogenic (14.63\%) and viral/ aseptic type (4\%).

Table-II

Etiological types and year wise distribution of meningitis cases $(n=326)$

\begin{tabular}{lcccc}
\hline Etiological Types ofmeningitis cases & \multicolumn{3}{c}{ Years } \\
\cline { 2 - 5 } & 2007 & 2008 & 2009 & Total \\
& No. $(\%)$ & No. $(\%)$ & No. $(\%)$ & No. $(\%)$ \\
\hline Tuberculous & $82(53.25)$ & $78(68.42)$ & $27(46.55)$ & $187(57.37)$ \\
Pyogenic & $58(37.66)$ & $33(28.95)$ & $22(37.93)$ & $113(34.66)$ \\
Viral/ Aseptic & $14(9.09)$ & $3(2.63)$ & $9(15.52)$ & $26(7.97)$ \\
\hline Total & $154(100)$ & $114(100)$ & $58(100)$ & $326(100)$ \\
\hline
\end{tabular}

Table-III

Distribution of the study population according to types of meningitis and nutritional status of the cases [n=224] Types of meningitis Undernutrition Difference of Undernutrition $\mathrm{Z} / \mathrm{p}$ Present Absent

\begin{tabular}{lllll}
\hline (a) Tuberculous meningitis ( $\mathrm{n}=127)$ & $96(75.59 \%)$ & $31(24.41 \%)$ & (a) vs. (b): & $3.06 /<0.01$ \\
(b) Pyogenic Meningitis $(\mathrm{n}=72)$ & $39(54.16 \%)$ & $33(45.83 \%)$ & (b) vs. (c): & $0.16 / 0.8728$ \\
(c) Viral/Aseptic Meningitis $(\mathrm{n}=25)$ & $14(56.00 \%)$ & $11(44.00 \%)$ & (c) Vs. (a): & $1.84 / 0.0658$ \\
\hline
\end{tabular}


Table-IV

Duration of hospital stay of meningitis cases $(n=224)$

\begin{tabular}{lccc}
\hline $\begin{array}{l}\text { Duration ofhospital } \\
\text { stay(Weeks) }\end{array}$ & $\begin{array}{c}\text { Tuberculous meningitis } \\
(\mathrm{n}=127)\end{array}$ & $\begin{array}{c}\text { Pyogenicmeningitis } \\
(\mathrm{n}=72)\end{array}$ & $\begin{array}{c}\text { Viral/Aseptic meningitis } \\
(\mathrm{n}=25)\end{array}$ \\
\hline$<1$ & No. $(\%)$ & No. $(\%)$ & $12(48.00 \%)$ \\
1 to 2 & $18(14.17 \%)$ & $23(28.05 \%)$ & $12(48.00 \%)$ \\
2 to 3 & $75(59.06 \%)$ & $47(57.32 \%)$ & $1(4.00 \%)$ \\
$>3$ & $27(21.26 \%)$ & $07(8.54 \%)$ & $0(0.00 \%)$ \\
\hline
\end{tabular}

Table-V

Distribution of the meningitis cases by etiological type and Case Fatality Rate (CFR) ( $n=326)$

\begin{tabular}{lcclc}
\hline Etiological Typesadmitted & Died & CFR & \multicolumn{2}{c}{ Difference of CFRZ / p } \\
\hline (a) Tuberculous meningitis $(n=187)$ & 60 & $32.08 \%$ & (a) vs (b): & $0.743 / 0.4592$ \\
(b) Pyogenic meningitis $(n=113)$ & 41 & $36.28 \%$ & (b) vs (c): & $5.534 /<0.001$ \\
(c) Viral/aseptic meningitis $(n=26)$ & 1 & $3.85 \%$ & (c) vs (a): & $5.546 /<0.001$ \\
\hline Total $(n=326)$ & 102 & $31.29 \%$ & &
\end{tabular}

Table - $V$ shows that the overall case fatality rate (CFR) in meningitis was $31.29 \%$. However, the CFR was highest in pyogenic meningitis $(36.28 \%)$, closely followed by tuberculous type (32.08\%). CFR was only $3.85 \%$ in case of viral/aseptic meningitis. Compared to viral meningitis, the CFR was significantly higher both in pyogenic $(p<0.001)$ and tuberculous meningitis $(p<0.001)$.

Figure-1 shows that some seasonal variation was there in different types of meningitis. For the pyogenic type two peaks were observed - in June and in September, for the tuberculous type (TBM) multiple peaks were observed - in January, March, May and August, whereas for viral/aseptic type such peaks were less conspicuous.

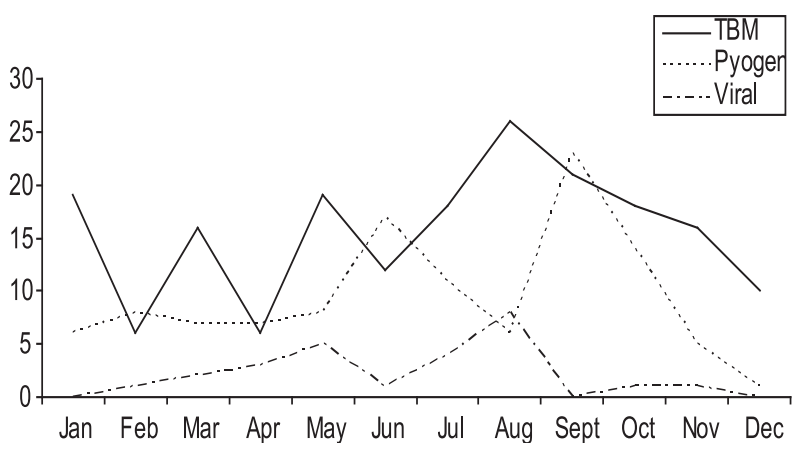

Fig.-1: Seasonal variation of meningitis cases (monthly cumulative numbers) ( $n=326)$

\section{Discussion}

The present study revealed that $44.17 \%$ of meningitis cases were below three years of age and the majority were males which were consistent with the study done by Amir et.al ${ }^{9}$. However, no sex difference in the attack rate of bacterial meningitis among children was observed in two other studies ${ }^{10,11}$.

By etiological type, $57.37 \%$ of the meningitis cases were tuberculous meningitis, $34.66 \%$ were of pyogenic and $7.97 \%$ were viral/aseptic origin. Contrary to our observation, in a hospital based study from 5 administrative areas of an Indian district (Vellore, Tamil Nadu) smaller proportion of bacterial meningitis $(18.56 \%)^{12}$ were reported. Smaller proportion of bacterial meningitis was also reported in another study ${ }^{13}$. These differences in proportions of different etiological types might be due to the differences in the age group of the study population, their race, ethnicity, case definition, nutritional status, health care seeking behavior, immunization coverage against the important bacterial agents, difference in hospital admission etc.

The present study found that overall $66.51 \%$ of the meningitis patients were undernourished. Elizabeth R. Reyes ${ }^{14}$ reported similar proportion of under nutrition among meningitis patients. In the present study undernutrition was found in significantly higher 
proportion of children with tuberculous meningitis compared to pyogenic cases $(p<0.01)$. Similar to our study Mitra et. al $^{15}$ also observed significant association between undernutrition and meningitis in Bangladesh. It suggests that under-nutrition might be a contributory factor of meningitis, though in another study no association between meningitis and malnutrition was noted ${ }^{16}$.

In the present study the overall Case Fatality Rate (CFR) was found to be $31.29 \%$. The CFR observed was highest among pyogenic meningitis (36.28\%), closely followed by Tuberculous meningitis (32.08\%); and only $3.85 \%$ in Viral/Aseptic meningitis. In a study in Pondicherry, South India, Sahai et.al ${ }^{17}$ found that case fatality rate was $25 \%$ among Bacterial meningitis cases. Contrary to our study, case fatality for bacterial meningitis varied between $2.2 \%$ to $9 \%$ as observed in different studies ${ }^{18,19,20}$. This differences might be due to differences in the study population, virulence of the infecting organisms, severity of cases, the time of initiation of treatment and the quality of care.

We observed in our study that in case of tuberculous meningitis there were four peaks spread over the year; in case of pyogenic meningitis two peaks in summer and post monsoon months; and in case of viral/aseptic type peaks were not much evident. Amir et.al ${ }^{9}$ noted seasonal variation in pyogenic meningitis with the peak in winter months; whereas for viral meningitis peak in the summer months were reported in different studies $^{21}$. This reflects that different pattern of seasonal variation of meningitis was observed in different studies. The reasons might be the differences of environmental conditions in different study areas.

Some variables could not be studied due to nonavailability of some records in this record based study. As it was a hospital based study, the information obtained do not reflect the actual situation in the community settings. So more studies (preferably community based studies or prospective studies in hospital settings) need to be conducted. This will enrich our knowledge and understanding on many areas, and thus help in reducing this important disease burden.

\section{Acknowledgement}

The authors express their gratefulness to the authority of Medical College \& Hospital, Kolkata for giving permission and extending help and cooperation in conducting the study.

\section{References}

1. Fellic JM K, Thomson AP. Long-term outcomes of childhood meningitis. Hosp Med 2002; 63:274-7.

2. Babiker MA, Taha SA. Meningitis in children of Riyadh. J Trop Med Hyg 1984; 87: 245-8.

3. Fortnum HM, Davis AC. Epidemiology of bacterial meningitis. Arch Dis Child 1993; 68: 763-7.

4. Parke JC Jr, Schneerson R, Robbin JB. The attack rate, age incidence, racial distribution and case fatality rate of Hemophilus influenzae type b meningitis in Mecklenberge County, North Carolina. J Pediatr 1972; 81: 765-9.

5. Bridger RC. Diagnosis and treatment of bacterial meningitis. Postgrad Doctor 1986; 9:282-7.

6. Agarwal DK. Physical and sexual growth pattern of affluent Indian children from 5 to 18 years of age. Indian Pediatr 1992; 29: 1203-82.

7. Agarwal DK. Physical growth in Indian sffluent children (Birth to 6 years). Indian Pediatr 1994; 21: 377-413.

8. SC Savva. Prevalence of sociodemographic associations of undernutrition and obesity among preschool children in Cyprus. European Journal of Clinical Nutrition 2005; 59: 1259-1265. [Available on www.nature.com ; accessed on 02 Oct. 2010].

9. Amir A, Zaheer M, Yunus M, Ahmad P, Ajmal M R. A clinico-epidemiological study of pyogenic meningitis in children. Indian J Matern Child Health 1993; 4(4):114-7.

10. Marji Sameer. Bacterial meningitis in children Rawal Med J 2007; 32:109-111.

11. Ashwal S, Parkin RM, Thompson JR. Bacterial meningitis in Children: current concepts of neurologic management. Adv Pediatr 1993; 40:185-215

12. Minz S, Balraj V, Lalitha M K, Murali N, Cherian T, Manoharan G, et al. Incidence of Haemophilus influenzae type b meningitis in India. Indian J Med Res 2008; 128: 57-64

13. Nigrovic L E, Kuppermann N, Malley R. Development and Validation of a Multivariable Predictive Model to Distinguish Bacterial from 
Aseptic Meningitis in Children in the PostHaemophilus influenzae Era. Pediatrics 2002; 110(4):712-18

14. Elizabeth R. Reyes. Suppurative Bacterial Meningitis: A6 years study. Phil J Microbiol Infect Dis 1986: 73-6.

15. Mitra AK, Albert MJ, Alam AN. Bacteraemia and meningitis among hospital patients with diarrhea. The transitions of the Royal Society of Tropical Medicine \& Hygiene 1993; 87(5): 560-3.

16. Rosen EU, Davis. Nutritional status of children with bacterial meningitis. S. Afr Med J 1980; 58:1004-6.

17. Sahai S, Mahadevan S, Srinivasan S, Kanungo R. Childhood bacterial meningitis in Pondicherry, South India. Indian J Pediatr. 2001; 68(9):83941.
18. Shaltout AA, Auger LT, Awadallah NB. Hijazi Z, Johny M, Hajj KE, et al. Morbidity and mortality of bacterial meningitis in Arab children. J Trop Med Hyg 1989; 92: 402-6.

19. Zaki M, Daoud AS, El Saleh Q, West PWJ. Childhood bacterial meningitis in Kuwait. J Trop Med Hyg 1990; 93: 7-11.

20. Qabazard Z, Ben-Nakhil HA, Badawi M, Haque E, Eliwa MS, Sharma PN. Childhood bacterial meningitis in Kuwait, incidence and etiology: a seven-year review of pediatric hospital admissions. Kuwait Medical Journal 2000; 32: 132-5.

21. Maguire HC, Atkinson $P$, Sharland M. Enterovirus infections in England and Wales : Laboratory surveillance data: 1975 to 1994 . Commun Dis Public Health 1999; 2:122-5. 